\title{
Response of Kharif Onion (Allium cepa L.) for Growth and Yield to Different Doses of Sulphur, GA 3 and NAA
}

\author{
Lal Singh $^{1 *}$, A. K. Barholia ${ }^{1}$, Rashmi Bajpai ${ }^{2}$, N.S. Bhadauria ${ }^{2}$ and V.B. Singh ${ }^{2}$ \\ ${ }^{1} R V S K V V$, Gwalior, (M.P.), India \\ ${ }^{2}$ College of Agriculture, Rajmata Vijyaraje Sciendia Krishi Vishwa Vidhyalaya, \\ Gwalior, India
}

*Corresponding author

\section{A B S T R A C T}

\begin{tabular}{l} 
Key w or d s \\
$\begin{array}{l}\text { Onion, Growth, } \\
\text { Yield, Quality, } \\
\text { Sulphur, Gibberellic } \\
\text { acid and NAA }\end{array}$ \\
\hline Article Info \\
$\begin{array}{l}\text { Accepted: } \\
\text { 15 December } 2018 \\
\text { Available Online: } \\
\text { 10 January } 2019\end{array}$ \\
\hline
\end{tabular}

The present investigation entitled "Response of Kharif onion (Allium cepa L.) for growth and yield to different doses of Sulphur, $\mathrm{GA}_{3}$ and NAA" was carried out during kharif 2016 -17 (first year) and 2017 - 18 (second year) at the experimental field RVSKVV, Krishi Vigyan Kendra, Rajgarh (M.P.) with 27 treatment combinations of three levels of sulphur i.e. 0,20 and $40 \mathrm{kgha}^{-1}$, three levels of Gibberellic acid viz., 0,50 and $100 \mathrm{ppm}$ and three levels of NAA i.e. 0, 50 and 100 ppm. Results obtained for 2016-17 and 2017-18 and in pooled basis revealed that the application of $40 \mathrm{~kg} \mathrm{~S} h a^{-1}$ was recorded significantly maximum plant height, number of leaves per plant, bulb/ green top ratio and neck diameter and yield attributes such as fresh weight of bulb, polar and equatorial diameter of bulb and bulb yield per hectare in both the year and in pooled. Foliar application of $\mathrm{GA}_{3} @ 50 \mathrm{ppm}$ $\left(\mathrm{G}_{1}\right)$ and NAA @ 100 PPM $\left(\mathrm{N}_{2}\right)$ at seedling stage and after 30 days after transplanting also exhibited significantly maximum growth and yield. It was concluded that various growth and yield parameters were improved with the application of S $40 \mathrm{Kg} / \mathrm{ha}+\mathrm{GA}_{3} 50 \mathrm{ppm}+$ NAA100ppm for higher yield and quality of Kharif Onion.

\section{Introduction}

Onion (Allium cepa L.) is one of the most important bulbous vegetable crops grown all over the world. The demand for onion is worldwide and it is found in most market of the world thought out the year. Onion is the oriented crop earning valuable foreign exchange for the country. It is an indispensable item in every kitchen and used to enhance flavour of different recipes. Onion has many medicinal values and used for preparation of various Homeopathic, Unani and Ayurvedic medicines. Researchers found that the more pungent onions exhibit strong anti-platelet activity. The production of kharif onion has several advantages i.e. increases total production per annum and fulfils the demand of fresh onion in the market. Kharif onion provides high price as compared to Rabi season crop. Application of sulphur not only increases the bulb yield but also improve its quality especially pungency and flavors. Sulphur deficient plants had poor utilization of nitrogen, phosphorus and potash. The translocation of food materials or for altering 
source to sink relationship is changed by application of plant growth regulators. Gibberellic acid stimulates cell division and elongation, germination of seeds, prevention of genetic dwarfism, increase flower and fruit size, dormancy and extending shelf life.

Naphthalene Acetic Acid (NAA) plays key role in cell elongation, cell division, vascular tissue differentiation, root initiation, apical dominance, leaf senescence, leaf and fruit abscission, fruit setting and flowering

\section{Materials and Methods}

The present investigation entitled "Response of Response of Kharif onion (Allium cepa L.) For growth and yield to different levelses of Sulphur, $\mathrm{GA}_{3}$ and NAA" was carried out during kharif 2016 - 17 (first year) and 2017 - 18 (second year) at the experimental field, Krishi Vigyan Kendra, Rajgarh (M.P.). The experimental material was comprised of 27 treatments combinations of three levels of sulphur i.e. 0, 20 and $40 \mathrm{~kg} \mathrm{ha}^{-1}$, three levels of Gibberellic acid viz., 0, 50 and 100PPM and three levels of NAA i.e. 0, 50 and 100 PPM. The foliar spray of plant growth regulators i.e. $\mathrm{GA}_{3}$ and NAA @ 50 and 100 ppm was done at seedling stage and after 30 DAT. Experiments were laid out in Factorial Randomized Complete Block Design with three replications. Observations were recorded on the basis of five random competitive plants selected from each treatment separately for growth and yield parameters and were evaluated as per standard procedure. The pooled data analysis was also performed

\section{Results and Discussion}

\section{Effect of sulphur on growth parameters}

Significantly maximum 60.01, 62.51 and $61.26 \mathrm{~cm}$ plant height was recorded under the treatment $\mathrm{S}_{2}\left(40 \mathrm{~kg} \mathrm{~S} \mathrm{ha}^{-1}\right)$ significantly followed by 58.07, 60.07 and 59.07 on plant height under the treatment $S_{1}\left(20 \mathrm{~kg} \mathrm{~S} \mathrm{ha}^{-1}\right)$ while, it was recorded lowest 52.73, 54.73 and $53.73 \mathrm{~cm}$ in treatment $S_{0}\left(0 \mathrm{~kg} \mathrm{~S} \mathrm{ha}^{-1}\right)$ at first year, second year and in pooled, respectively (Table 1 ). Similarly significantly maximum 12.64, 13.34 and 12.99 leaves per plant was recorded under the treatment $S_{2}$ $\left(40 \mathrm{~kg} \mathrm{~S} \mathrm{ha}^{-1}\right)$ significantly followed by 12.11 , 12.61 and 12.36 leaves per plant under the treatment $\mathrm{S}_{1}\left(20 \mathrm{~kg} \mathrm{~S} \mathrm{ha}^{-1}\right)$ while, it was recorded lowest $10.43,10.93$ and 10.68 in treatment $\mathrm{S}_{0}\left(0 \mathrm{~kg} \mathrm{~S} \mathrm{ha}^{-1}\right)$ at first year, second year and in pooled, respectively. The significantly maximum $1.46,2.00$ and 1.73 $\mathrm{bulb} /$ green top ratio was registered under the treatment $S_{2}\left(40 \mathrm{~kg} \mathrm{~S} \mathrm{ha}^{-1}\right)$ it was significantly followed by $1.36,1.68$ and 1.53 bulb/green top ratio under $S_{1}\left(20 \mathrm{~kg} \mathrm{~S} \mathrm{ha}^{-1}\right)$, while, it was noted lowest 1.20, 1.50 and 1.35 in treatment $\mathrm{S}_{0}\left(0 \mathrm{~kg} \mathrm{~S} \mathrm{ha}^{-1}\right)$ at first year, second year and in pooled, respectively. Significantly maximum 1.36, 1.85 and $1.60 \mathrm{~cm}$ neck diameter was recorded under the treatment $S_{2}$ $\left(40 \mathrm{~kg} \mathrm{~S} \mathrm{ha}^{-1}\right)$, while, it was noted lowest 0.85 , 1.15 and $1.00 \mathrm{~cm}$ in treatment $S_{0}\left(0 \mathrm{~kg} \mathrm{~S} \mathrm{ha}^{-1}\right)$ at first year, second year and in pooled, respectively. Application of S $20 \mathrm{~kg} / \mathrm{ha}^{-1}$ (S1) was found non- significant to treatment $\mathrm{S}_{2}$ $\left(40 \mathrm{~kg} \mathrm{~S} \mathrm{ha}^{-1}\right)$ (Table 1). The present results are therefore in conformity with the results of Suman et al., (2002), Rashid (2010), Jain et al., (2014) and El Sayed et al., (2015). Plant height, number of leaves per plant, bulb/ green top ratio and neck diameter significantly increased with increasing sulphur levels up to $40 \mathrm{~kg} \mathrm{~S} \mathrm{ha}^{-1}$. These results may be due to the beneficial effect of sulphur application as an one of the required elements for plant growth, it is important in protein and chlorophyll formation.

\section{Effect of $\mathbf{G A}_{3}$ on growth parameters}

The treatment $\mathrm{G}_{1} \quad(50 \mathrm{ppm})$ was found significantly superior as compared to $G_{2}(100$ ppm) treatments. Treatment $\mathrm{G}_{1}(50 \mathrm{ppm})$ was 
recorded significantly maximum plant height $57.63,59.87$ and $58.75 \mathrm{~cm}$ (Table 1), while, it was recorded lowest 56.14, 58.23 and 57.19 $\mathrm{cm}$ in treatment $\mathrm{G}_{0}(0 \mathrm{ppm}$ i.e. control $)$ at first year, second year and in pooled, respectively. This result was in harmony with those reported by Anwar (2001), Singh et al., (2014), Govind et al., (2015), Trivedi and Dhumal (2017), and Yadagiri et al., (2018). The treatment $\mathrm{G}_{1}$ (50ppm) was recorded significantly maximum 11.96, 12.56 and 12.26 leaves per plant which was at par with $\mathrm{G}_{2}$ (100ppm) (11.77, 12.33 and 12.05 leaves per plant), while, it was recorded lowest 11.46, 11.99 and 11.73 in treatment $\mathrm{G}_{0}(0$ ppm i.e. control) at first year, second year and in pooled, respectively. Treatment $\mathrm{G}_{1}$ $(50 \mathrm{ppm})$ exhibited significantly maximum $1.38,1.78$ and $1.58 \mathrm{bulb} /$ green top ratio at par with $\mathrm{G}_{2}$ (100ppm) (1.35, 1.76 and 1.56), while, it was recorded lowest 1.31, 1.64 and 1.48 in treatment $G_{0}(0 \mathrm{ppm}$ i.e. control $)$ at first year, second year and in pooled, respectively.

The treatment $\mathrm{G}_{1} \quad(50 \mathrm{ppm})$ exhibited significantly maximum $1.22,1.62$ and 1.42 $\mathrm{cm}$ neck diameter which was at par with $\mathrm{G}_{2}$ (100 ppm), while, it was recorded lowest $1.11,1.43$ and $1.27 \mathrm{~cm}$ in treatment $\mathrm{G}_{0}(0$ ppm i.e. control) at first year, second year and in pooled, respectively. The results obtained are in conformity with the results of Anwar (2001), Singh et al., (2014), Govind et al., (2015) and Yadagiri et al., (2018). The improving plant growth under spraying of gibberellic acid may be due to the role of gibberellic acid on enhancing cell division activity, increasing of proline accumulation of plant and increasing of endogenous phytohormones i.e. increasing promotion hormones (IAA, $\mathrm{GA}_{3}$ and cytokinins) and reducing $\mathrm{ABA}$ content, which found that plant growth regulators make a shift in hormonal balance characterized by increase in endogenous phytohormon in plant.

\section{Effect of NAA on growth parameters}

Significantly maximum 58.34, 60.62 and $59.48 \mathrm{~cm}$ plant height was recorded under the treatment $\mathrm{N}_{2}$ (NAA $100 \mathrm{PPM}$ ) at par with $\mathrm{N}_{1}$ (NAA 50 PPM) (57.90, 57.90 and $60.12 \mathrm{~cm}$ ), while, it was recorded lowest 54.56, 56.56 and $55.56 \mathrm{~cm}$ in treatment $\mathrm{N}_{0}$ (NAA 0 PPM) at first year, second year and in pooled, respectively. Similarly significantly maximum 12.10, 12.71 and 12.41 leaves per plant was recorded under the treatment $\mathrm{N}_{2}$ (NAA 100 PPM) at par with $\mathrm{N}_{1}$ (NAA 50 PPM) (12.0, 12.58 and 12.29 leaves per plant), while, it was recorded lowest 11.09, 11.59 and 11.34 in treatment $\mathrm{N}_{0}$ (NAA 0 PPM) at first year, second year and in pooled, respectively. Among NAA, significantly maximum 1.40, 1.82 and $1.61 \mathrm{bulb} /$ green top ratio was noticed under the treatment $\mathrm{N}_{2}$ (NAA 100 PPM) which was at par with $\mathrm{N}_{1}$ (NAA 50 PPM) (1.38, 1.81 and 1.60), while, it was observed lowest 1.26, 1.56 and 1.41 in treatment $\mathrm{N}_{0}$ (NAA 0 PPM) at first year, second year and in pooled, respectively. Significantly maximum $1.23,1.65$ and 1.44 $\mathrm{cm}$ neck diameter was noticed under the treatment $\mathrm{N}_{2}$ (NAA 100 PPM) followed by $\mathrm{N}_{1}$ (NAA 50 PPM) $(1.20,1.58$ and $1.39 \mathrm{~cm})$ at par in first year only, while, it was observed lowest $1.05,1.35$ and $1.20 \mathrm{~cm}$ in treatment $\mathrm{N}_{0}$ (NAA 0 PPM) at first, second year and pooled, respectively.

The present results are therefore in conformity with the results of Bose et al., (2009), Meena et al., (2017) and Pratap et al., (2017). The increase in plant height, leaves per plant, bulb/ green top ratio and neck diameter by foliar spray of NAA 100PPM might be due to rapid increase in cell division and cell elongation in the meristemic region. In general, leaf is considered as an important functional unit of plant which contributes to yield. Probable reason may be due to the role of NAA on enhancing cell division activity, 
increasing proline accumulation of plant and increasing endogenous phytohormones.

\section{Interaction effect of sulphur, $\mathrm{GA}_{3}$ and NAA on growth parameters}

The maximum 63.60, 66.77 and $65.18 \mathrm{~cm}$ plant height were recorded in treatment combination $\mathrm{S}_{2} \mathrm{G}_{1} \mathrm{~N}_{2}$ (S $40 \mathrm{Kg} / \mathrm{ha}+\mathrm{GA}_{3}$ 50ppm+ NAA100ppm) followed by $\mathrm{S}_{2} \mathrm{G}_{1} \mathrm{~N}_{1}$ (S $40 \mathrm{Kg} / \mathrm{ha}+\mathrm{GA}_{3}$ 50ppm+ NAA50ppm) $(61.93,64.93$ and $63.43 \mathrm{~cm})$, while, it was recorded lowest $50.27,52.27$ and $51.27 \mathrm{~cm}$ in treatment $\mathrm{S}_{0} \mathrm{G}_{0} \mathrm{~N}_{0}\left(\mathrm{~S} 0 \mathrm{Kg} / \mathrm{ha}+\mathrm{GA}_{3} 0 \mathrm{ppm}+\right.$ NAA 0ppm) at first year, second year and in pooled, respectively (Table 2). The maximum 13.43, 14.43 and 13.93 leaves per plant were recorded in treatment combination $\mathrm{S}_{2} \mathrm{G}_{1} \mathrm{~N}_{2}$ (S $40 \mathrm{Kg} / \mathrm{ha}+\mathrm{GA}_{3}$ 50ppm+ NAA100ppm) followed by $\mathrm{S}_{2} \mathrm{G}_{1} \mathrm{~N}_{1}$ (S $40 \mathrm{Kg} / \mathrm{ha}+\mathrm{GA}_{3}$ 50ppm+ NAA50ppm) (13.33, 14.25 and 13.79), while, it was recorded lowest 9.70, 10.20 and 9.95 in treatment $\mathrm{S}_{0} \mathrm{G}_{0} \mathrm{~N}_{0}(\mathrm{~S} 0$ $\mathrm{Kg} / \mathrm{ha}+\mathrm{GA}_{3}$ Oppm+ NAA 0ppm) at first year, second year and in pooled, respectively. Similarly significantly maximum 1.57, 2.37 and $1.97 \mathrm{bulb} /$ green top ratio were recorded in treatment combination $\mathrm{S}_{2} \mathrm{G}_{1} \mathrm{~N}_{2}$ (S 40 $\mathrm{Kg} / \mathrm{ha}+\mathrm{GA}_{3}$ 50ppm+ NAA100ppm) followed by $\mathrm{S}_{2} \mathrm{G}_{1} \mathrm{~N}_{1} \quad\left(\mathrm{~S} 40 \mathrm{Kg} / \mathrm{ha}+\mathrm{GA}_{3} \quad 50 \mathrm{ppm}+\right.$ NAA50ppm) (1.55, 2.26 and 1.91), while, it was recorded lowest $1.13,1.43$ and 1.28 in treatment $\mathrm{S}_{0} \mathrm{G}_{0} \mathrm{~N}_{0}\left(\mathrm{~S} 0 \mathrm{Kg} / \mathrm{ha}+\mathrm{GA}_{3} 0 \mathrm{ppm}+\right.$ NAA 0ppm) at first, second year and pooled, respectively. Significantly maximum 1.51, 2.31 and $1.91 \mathrm{~cm}$ neck diameter were recorded in treatment combination $\mathrm{S}_{2} \mathrm{G}_{1} \mathrm{~N}_{2}(\mathrm{~S}$ $\left.40 \mathrm{Kg} / \mathrm{ha}+\mathrm{GA}_{3} 50 \mathrm{ppm}+\mathrm{NAA} 100 \mathrm{ppm}\right)$ followed by $\mathrm{S}_{2} \mathrm{G}_{1} \mathrm{~N}_{1}$ (S $40 \mathrm{Kg} / \mathrm{ha}+\mathrm{GA}_{3}$ 50ppm+ NAA50ppm) (1.46, 2.18 and 1.82 $\mathrm{cm})$, while, it was recorded lowest $0.69,0.99$ and $0.84 \mathrm{~cm}$ in treatment $\mathrm{S}_{0} \mathrm{G}_{0} \mathrm{~N}_{0}(\mathrm{~S} 0 \mathrm{Kg} / \mathrm{ha}+$ $\mathrm{GA}_{3}$ Oppm+ NAA 0ppm) at first, second year and pooled, respectively. Similar finding were also rep[orted by Sitapara et al., (2011) and Meena et al., (2017) Plant height is a genetically controlled character but several studies have indicated that the plant height can be either increased or decreased by the application of synthetic plant growth regulators and sulphur. Probably may be due to the beneficial cumulative effect of sulphur, $\mathrm{GA}_{3}$ and NAA. Application of sulphur as a one of the required elements for plant growth, it is important in protein and chlorophyll formation. The increase in plant height, number of leaves per plant, bulb/ green top ratio and neck diameter by foliar spray of $\mathrm{GA}_{3}$ and NAA might be due to rapid increase in cell division and cell elongation in the meristemic region. The thickness of the stem (neck) is the important parameter for storage of bulb. Hence, more the thickness of the neck, more will be the rotting due to more fungous infection.

\section{Effect of sulphur on yield and yield parameters}

Significantly maximum 90.93, 96.46 and 93.69 $\mathrm{g}$ fresh weight of bulb was recorded under the treatment $S_{2}\left(40 \mathrm{~kg} \mathrm{~S} \mathrm{ha}{ }^{-1}\right)$, which was significantly superior to $\mathrm{S}_{1}\left(20 \mathrm{~kg} \mathrm{~S}\right.$ ha $^{-}$ ${ }^{1}$ )while, it was noted lowest 74.32, 79.36 and $76.84 \mathrm{~g}$ in treatment $\mathrm{S}_{0}\left(0 \mathrm{~kg} \mathrm{~S} \mathrm{ha}{ }^{-1}\right)$ at first year, second year and in pooled, respectively (Table 3). The Polar diameter of bulb was recorded significantly maximum 5.89, 6.39 and $6.14 \mathrm{~cm}$ under the treatment $S_{2}(40 \mathrm{~kg} S$ $\mathrm{ha}^{-1}$ ) which was significantly superior to $S_{1}$ $\left(20 \mathrm{~kg} \mathrm{~S} \mathrm{ha}^{-1}\right)$ while, it was noted lowest 5.23 , 5.53 and $5.38 \mathrm{~cm}$ in treatment $\mathrm{S}_{0}\left(0 \mathrm{~kg} \mathrm{~S} \mathrm{ha}^{-1}\right)$ at first, second year and pooled, respectively. Equatorial diameter of bulb was recorded significantly maximum 4.82, 5.26 and 5.04 $\mathrm{cm}$ under the treatment $S_{2}\left(40 \mathrm{~kg} \mathrm{~S} \mathrm{ha}^{-1}\right)$ whish was significantly superior to treatment S1 (20 $\left.\mathrm{kg} \mathrm{S} \mathrm{ha}{ }^{-1}\right)$ and it was noted lowest 4.35, 4.65 and $4.50 \mathrm{~cm}$ in treatment $S_{0}\left(0 \mathrm{~kg} \mathrm{~S} \mathrm{ha}^{-1}\right)$ at first year, second year and in pooled, respectively. The treatment $S_{2}\left(40 \mathrm{~kg} \mathrm{~S} \mathrm{ha}^{-1}\right)$ was recorded significantly maximum 244.01, 240.31 and $242.16 \mathrm{q} / \mathrm{ha}$ bulb yield and it was noted lowest 198.17, 189.17 and $193.67 \mathrm{q} / \mathrm{ha}$ 
in treatment $S_{0}\left(0 \mathrm{~kg} \mathrm{~S} \mathrm{ha}^{-1}\right)$ at first year, second year and in pooled, respectively. Similar results were also reported in onion crop by Suman et al., (2002), Rashid (2010), Jain et al., (2014) and El Sayed et al., (2015). The increase in fresh weight of bulb, polar and equatorial diameter of bulb and bulb yield might be attributed to the increased synthesis of sulphur containing amino acids in plants which intern resulted in the formation of healthy Xylem, collenchyma and schlernchyma tissues. It also increased the uptake of $\mathrm{N}, \mathrm{P}, \mathrm{K}$ and $\mathrm{S}$ which might have influenced the synthesis and translocation of stored materials to the sink.

\section{Effect of $\mathrm{GA}_{3}$ on yield and yield parameters}

Treatment $\mathrm{G}_{1} \quad\left(\mathrm{GA}_{3} @\right.$ $@$ 50ppm) exhibited significantly maximum 85.69, 90.93 and $88.31 \mathrm{~g}$ fresh weight of bulb, which was significantly inferior too treatment $\mathrm{G}_{2}\left(\mathrm{GA}_{3} @\right.$ $100 \mathrm{ppm}$ ) while, it was recorded lowest 81.25 , 86.46 and $83.85 \mathrm{~g}$ in treatment $\mathrm{G}_{0}(0 \mathrm{ppm}$ i.e. control) at first, second year and pooled, respectively. The treatment $\mathrm{G}_{1} \quad\left(\mathrm{GA}_{3}\right.$ @ $50 \mathrm{ppm}$ ) exhibited significantly maximum $5.69,6.09$ and $5.89 \mathrm{~cm}$ polar diameter of bulb, followed by treatment of $\mathrm{G}_{2}\left(\mathrm{GA}_{3} @ 100 \mathrm{ppm}\right)$ while, it was recorded lowest 5.52, 5.85 and $5.69 \mathrm{~cm}$ in treatment $\mathrm{G}_{0}(0 \mathrm{ppm}$ i.e. control $)$ at first year, second year and in pooled, respectively. Similar results have also been reported by Rashid (2010), Singh et al., (2014) and Yadagiri et al., (2018). Foliar application of $\mathrm{GA}_{3} @ 50 \mathrm{ppm}\left(\mathrm{G}_{1}\right)$ was exhibited significantly maximum 4.69, 5.11 and $4.90 \mathrm{~cm}$ equatorial diameter of bulb followed by treatment $\mathrm{G}_{2}\left(\mathrm{GA}_{3} @ 100 \mathrm{ppm}\right)$ while, it was recorded lowest 4.56, 4.89 and $4.73 \mathrm{~cm}$ in treatment $\mathrm{G}_{0}(0 \mathrm{ppm}$ i.e. control $)$ at first, second year and pooled, respectively. Foliar application of $\mathrm{GA}_{3} @ 50 \mathrm{ppm}\left(\mathrm{G}_{1}\right)$ exhibited significantly maximum 231.01, 224.53 and $227.77 \mathrm{q} / \mathrm{ha}$ bulb yield which was significantly while which was significantly followed by treatment $\mathrm{G} 2\left(\mathrm{GA}_{3} @ 100 \mathrm{ppm}\right)$ it was recorded lowest 216.73, 208.62 and $212.67 \mathrm{q} / \mathrm{ha}$ in treatment $\mathrm{G}_{0}(0 \mathrm{ppm}$ i.e. control) at first year, second year and in pooled, respectively. Similar results were also reported by Rashid (2010), Singh et al., (2014), Govind et al., (2015), Yadagiri et al., (2018) and Thakur et al., (2018). Fresh weight of bulb, polar and equatorial diameter of bulb showed upward trend with the increase in $\mathrm{GA}_{3}$ concentrations which could be due to the rapid cell division and elongation leading to bigger bulb formation. It could be concluded that the heaviest bulbs yield which resulted may be attributed to the best vigour of plant growth characters which obtained by addition of foliar application of 50PPM GA $\mathrm{G}_{3}$. There is no doubt that, growth regulators play a major role in diverse growth processes including organ elongation and senescence.

\section{Effect of NAA on yield and yield parameters}

Significantly maximum 86.73, 92.01 and $89.37 \mathrm{~g}$ fresh weight of bulb was noticed under the treatment $\mathrm{N}_{2}$ (NAA 100 PPM) significantly followed by $\mathrm{N}_{1}$ (NAA $50 \mathrm{ppm}$ ) while, it was observed lowest 78.11, 83.11 and $80.61 \mathrm{~g}$ in treatment $\mathrm{N}_{0}$ (NAA 0 PPM) at first year, second year and in pooled, respectively. Among NAA, significantly maximum 5.73, 6.14 and $5.94 \mathrm{~cm}$ polar diameter of bulb was noticed under the treatment $\mathrm{N}_{2}$ (NAA 100 PPM), followed by $\mathrm{N}_{1}$ (NAA @ $50 \mathrm{ppm}$ ) while, it was observed lowest $5.41,5.71$ and $5.56 \mathrm{~cm}$ in treatment $\mathrm{N}_{0}$ (NAA 0 PPM) at first year, second year and in pooled, respectively. Significantly maximum 4.70, 5.08 and $4.89 \mathrm{~cm}$ equatorial diameter of bulb was noticed under the treatment $\mathrm{N}_{2}$ (NAA 100 PPM) followed by $\mathrm{N}_{1}$ (NAA @ 50 ppm) while, it was observed lowest $4.51,4.84$ and $4.68 \mathrm{~cm}$ in treatment $\mathrm{N}_{0}$ (NAA @0 PPM) at first year, second year and in pooled, respectively. 
Table.1 Effect of different doses of Sulphur, $\mathrm{GA}_{3}$ and NAA on growth at harvest in first, second year and pooled

\begin{tabular}{|c|c|c|c|c|c|c|c|c|c|c|c|c|c|}
\hline \multirow{2}{*}{$\begin{array}{l}\text { Treat. } \\
\text { Symb. }\end{array}$} & \multirow[t]{2}{*}{ Treatments } & \multicolumn{3}{|c|}{ Plant height (cm) } & \multicolumn{3}{|c|}{ No. of leaves/plant } & \multicolumn{3}{|c|}{ Bulb/green top ratio } & \multicolumn{3}{|c|}{ Neck diameter (cm) } \\
\hline & & $\begin{array}{c}1^{\text {st }} \\
\text { Year }\end{array}$ & $\begin{array}{l}2^{\text {nd }} \\
\text { Year }\end{array}$ & Pooled & $\begin{array}{c}1^{\text {st }} \\
\text { Year }\end{array}$ & $\begin{array}{c}2^{\text {nd }} \\
\text { Year }\end{array}$ & Pooled & $\begin{array}{c}1^{\text {st }} \\
\text { Year }\end{array}$ & $\begin{array}{c}2^{\text {nd }} \\
\text { Year }\end{array}$ & Pooled & $\begin{array}{c}1^{\text {st }} \\
\text { Year }\end{array}$ & $\begin{array}{c}2^{\text {nd }} \\
\text { Year }\end{array}$ & Pooled \\
\hline $\mathbf{S}_{\mathbf{0}}$ & Sulphur (0 kg/ha) & 52.73 & 54.73 & 53.73 & 10.43 & 10.93 & 10.68 & 1.20 & 1.50 & 1.35 & 0.85 & 1.15 & 1.00 \\
\hline $\mathbf{S}_{1}$ & Sulphur (20 kg/ha) & 58.07 & 60.07 & 59.07 & 12.11 & 12.61 & 12.36 & 1.38 & 1.68 & 1.53 & 1.27 & 1.57 & 1.42 \\
\hline \multirow{3}{*}{$\mathbf{S}_{2}$} & Sulphur (40 kg/ha) & 60.01 & 62.51 & 61.26 & 12.64 & 13.34 & 12.99 & 1.46 & 2.00 & 1.73 & 1.36 & 1.85 & 1.60 \\
\hline & S.Em \pm & 0.41 & 0.23 & 0.21 & 0.07 & 0.05 & 0.04 & 0.02 & 0.02 & 0.02 & 0.015 & 0.019 & 0.015 \\
\hline & C.D. (P 0.05) le & 1.18 & 0.65 & 0.60 & 0.19 & 0.15 & 0.11 & 0.05 & 0.05 & 0.05 & 0.042 & 0.054 & 0.042 \\
\hline $\mathbf{G}_{\mathbf{0}}$ & $\mathrm{GA}_{3}(0 \mathrm{PPM})$ & 56.14 & 58.23 & 57.19 & 11.46 & 11.99 & 11.73 & 1.31 & 1.64 & 1.48 & 1.11 & 1.43 & 1.27 \\
\hline $\mathbf{G}_{1}$ & $\mathrm{GA}_{3}(50 \mathrm{PPM})$ & 57.63 & 59.87 & 58.75 & 11.96 & 12.56 & 12.26 & 1.38 & 1.78 & 1.58 & 1.22 & 1.62 & 1.42 \\
\hline \multirow[t]{3}{*}{$\mathbf{G}_{2}$} & $\mathrm{GA}_{3}(100 \mathrm{PPM})$ & 57.03 & 59.19 & 58.11 & 11.77 & 12.33 & 12.05 & 1.35 & 1.76 & 1.56 & 1.15 & 1.52 & 1.34 \\
\hline & S.Em \pm & 0.41 & 0.23 & 0.21 & 0.07 & 0.05 & 0.04 & 0.02 & 0.02 & 0.02 & 0.015 & 0.019 & 0.015 \\
\hline & C.D. (P 0.0 & 1.18 & 0.65 & 0.60 & 0.19 & 0.15 & 0.11 & 0.05 & 0.05 & 0.05 & 0.042 & 0.054 & 0.042 \\
\hline $\mathbf{N}_{0}$ & NAA (0 PPM) & 54.56 & 56.56 & 55.56 & 11.09 & 11.59 & 11.34 & 1.26 & 1.56 & 1.41 & 1.05 & 1.35 & 1.20 \\
\hline $\mathbf{N}_{1}$ & NAA (50 PPM) & 57.90 & 60.12 & 59.01 & 12.00 & 12.58 & 12.29 & 1.38 & 1.81 & 1.60 & 1.20 & 1.58 & 1.39 \\
\hline \multirow[t]{3}{*}{$\mathbf{N}_{2}$} & NAA (100 PPM) & 58.34 & 60.62 & 59.48 & 12.10 & 12.71 & 12.41 & 1.40 & 1.82 & 1.61 & 1.23 & 1.65 & 1.44 \\
\hline & S.Em \pm & 0.41 & 0.23 & 0.21 & 0.07 & 0.05 & 0.04 & 0.02 & 0.02 & 0.02 & 0.85 & 1.15 & 1.00 \\
\hline & C.D. (P 0.05) level & 1.18 & 0.65 & 0.60 & 0.19 & 0.15 & 0.11 & 0.05 & 0.05 & 0.05 & 1.27 & 1.57 & 1.42 \\
\hline
\end{tabular}


Table.2 Interaction effect of different doses of Sulphur, $\mathrm{GA}_{3}$ and NAA on growth at harvest in first year, second year and in pooled basis

\begin{tabular}{|c|c|c|c|c|c|c|c|c|c|c|c|c|c|}
\hline \multirow{2}{*}{$\begin{array}{l}\text { Treat. } \\
\text { Symb. }\end{array}$} & \multirow[t]{2}{*}{ Treatments } & \multicolumn{3}{|c|}{ Plant height (cm) } & \multicolumn{3}{|c|}{ No. of leaves/plant } & \multicolumn{3}{|c|}{ Bulb/green top ratio } & \multicolumn{3}{|c|}{ Neck diameter $(\mathbf{c m})$} \\
\hline & & $1^{\text {st }}$ Year & $2^{\text {nd }}$ Year & Pooled & $1^{\text {st }}$ Year & $2^{\text {nd }}$ Year & Pooled & $1^{\text {st }}$ Year & $2^{\text {nd }}$ Year & Pooled & $1^{\text {st }}$ Year & $2^{\text {nd }}$ Year & Pooled \\
\hline $\mathbf{S}_{0} \mathbf{G}_{0} \mathbf{N}_{0}$ & $\mathrm{~S}(0 \mathrm{Kg} / \mathrm{ha}) \mathrm{G}(0 \mathrm{ppm}) \mathrm{N}(0 \mathrm{ppm})$ & 50.27 & 52.27 & 51.27 & 9.70 & 10.20 & 9.95 & 1.13 & 1.43 & 1.28 & 0.69 & 0.99 & 0.84 \\
\hline $\mathrm{S}_{0} \mathrm{G}_{0} \mathrm{~N}_{1}$ & $\mathrm{~S}(0 \mathrm{Kg} / \mathrm{ha}) \mathrm{G}(0 \mathrm{ppm}) \mathrm{N}(50 \mathrm{ppm})$ & 53.30 & 55.30 & 54.30 & 10.17 & 10.67 & 10.42 & 1.19 & 1.49 & 1.34 & 0.80 & 1.10 & 0.95 \\
\hline $\mathbf{S}_{0} \mathbf{G}_{0} \mathbf{N}_{2}$ & $\mathrm{~S}(0 \mathrm{Kg} / \mathrm{ha}) \mathrm{G}(0 \mathrm{ppm}) \mathrm{N}(100 \mathrm{ppm})$ & 53.60 & 55.60 & 54.60 & 10.37 & 10.87 & 10.62 & 1.20 & 1.50 & 1.35 & 0.85 & 1.15 & 1.00 \\
\hline $\mathbf{S}_{0} \mathbf{G}_{1} \mathbf{N}_{0}$ & $\mathrm{~S}(0 \mathrm{Kg} / \mathrm{ha}) \mathrm{G}(50 \mathrm{ppm}) \mathrm{N}(0 \mathrm{ppm})$ & 51.33 & 53.33 & 52.33 & 10.07 & 10.57 & 10.32 & 1.17 & 1.47 & 1.32 & 0.76 & 1.06 & 0.91 \\
\hline $\mathrm{S}_{0} \mathbf{G}_{1} \mathbf{N}_{1}$ & $\mathrm{~S}(0 \mathrm{Kg} / \mathrm{ha}) \mathrm{G}(50 \mathrm{ppm}) \mathrm{N}(50 \mathrm{ppm})$ & 53.87 & 55.87 & 54.87 & 11.00 & 11.50 & 11.25 & 1.25 & 1.55 & 1.40 & 0.98 & 1.28 & 1.13 \\
\hline $\mathrm{S}_{0} \mathrm{G}_{1} \mathrm{~N}_{2}$ & $\mathrm{~S}(0 \mathrm{Kg} / \mathrm{ha}) \mathrm{G}(50 \mathrm{ppm}) \mathrm{N}(100 \mathrm{ppm})$ & 54.30 & 56.30 & 55.30 & 11.03 & 11.53 & 11.28 & 1.27 & 1.57 & 1.42 & 1.11 & 1.41 & 1.26 \\
\hline $\mathbf{S}_{0} \mathbf{G}_{2} \mathbf{N}_{0}$ & $\mathrm{~S}(0 \mathrm{Kg} / \mathrm{ha}) \mathrm{G}(100 \mathrm{ppm}) \mathrm{N}(0 \mathrm{ppm})$ & 50.47 & 52.47 & 51.47 & 9.93 & 10.43 & 10.18 & 1.15 & 1.45 & 1.30 & 0.72 & 1.02 & 0.87 \\
\hline $\mathrm{S}_{0} \mathrm{G}_{2} \mathrm{~N}_{1}$ & $\mathrm{~S}(0 \mathrm{Kg} / \mathrm{ha}) \mathrm{G}(100 \mathrm{ppm}) \mathrm{N}(50 \mathrm{ppm})$ & 53.60 & 55.60 & 54.60 & 10.67 & 11.17 & 10.92 & 1.22 & 1.52 & 1.37 & 0.87 & 1.17 & .02 \\
\hline $\mathrm{S}_{0} \mathrm{G}_{2} \mathrm{~N}_{2}$ & $\mathrm{~S}(0 \mathrm{Kg} / \mathrm{ha}) \mathrm{G}(100 \mathrm{ppm}) \mathrm{N}(100 \mathrm{ppm})$ & 53.80 & 55.80 & 54.80 & 10.97 & 11.47 & 11.22 & 1.23 & 1.53 & 1.38 & 0.90 & 1.20 & 1.05 \\
\hline$S_{1} G_{0} N_{0}$ & $\mathrm{~S}(20 \mathrm{Kg} / \mathrm{ha}) \mathrm{G}(0 \mathrm{ppm}) \mathrm{N}(0 \mathrm{ppm})$ & 54.80 & 56.80 & 55.80 & 11.57 & 12.07 & 11.82 & 1.28 & 1.58 & 1.43 & 1.16 & 1.46 & 1.31 \\
\hline $\mathrm{S}_{1} \mathbf{G}_{0} \mathbf{N}_{1}$ & $\mathrm{~S}(20 \mathrm{Kg} / \mathrm{ha}) \mathrm{G}(0 \mathrm{ppm}) \mathrm{N}(50 \mathrm{ppm})$ & 58.27 & 60.27 & 59.27 & 12.10 & 12.60 & 12.35 & 1.37 & 1.67 & 1.52 & 1.28 & 1.58 & 1.43 \\
\hline $\mathrm{S}_{1} \mathrm{G}_{0} \mathrm{~N}_{2}$ & $\mathrm{~S}(20 \mathrm{Kg} / \mathrm{ha}) \mathrm{G}(0 \mathrm{ppm}) \mathrm{N}(100 \mathrm{ppm})$ & 59.17 & 61.17 & 60.17 & 12.13 & 12.63 & & 1.40 & 0 & 5 & .28 & 1.58 & 43 \\
\hline $\mathbf{S}_{1} \mathbf{G}_{1} \mathbf{N}_{0}$ & $\mathrm{~S}(20 \mathrm{Kg} / \mathrm{ha}) \mathrm{G}(50 \mathrm{ppm}) \mathrm{N}(0 \mathrm{ppm})$ & 56.73 & 58.73 & 57.73 & 11.67 & 12.17 & 11.92 & 1.32 & 1.62 & 1.47 & 1.20 & 1.50 & 1.35 \\
\hline $\mathbf{S}_{1} \mathbf{G}_{1} \mathbf{N}_{1}$ & $\mathrm{~S}(20 \mathrm{Kg} / \mathrm{ha}) \mathrm{G}(50 \mathrm{ppm}) \mathrm{N}(50 \mathrm{ppm})$ & 59.27 & 61.27 & 60.27 & 12.60 & 13.10 & 12.85 & 1.45 & 1.75 & 1.60 & 1.34 & 1.64 & 1.49 \\
\hline $\mathrm{S}_{1} \mathbf{G}_{1} \mathbf{N}_{2}$ & $\mathrm{~S}(20 \mathrm{Kg} / \mathrm{ha}) \mathrm{G}(50 \mathrm{ppm}) \mathrm{N}(100 \mathrm{ppm})$ & 59.40 & 61.40 & 60.40 & 12.67 & 13.17 & 12.92 & 1.46 & 1.76 & 1.61 & 1.35 & 1.65 & 1.50 \\
\hline $\mathrm{S}_{1} \mathbf{G}_{2} \mathbf{N}_{0}$ & $\mathrm{~S}(20 \mathrm{Kg} / \mathrm{ha}) \mathrm{G}(100 \mathrm{ppm}) \mathrm{N}(0 \mathrm{ppm})$ & 56.63 & 58.63 & 57.63 & 11.63 & 12.13 & 11.88 & 1.30 & 1.60 & 1.45 & 1.17 & 1.47 & 1.32 \\
\hline $\mathbf{S}_{1} \mathbf{G}_{2} \mathbf{N}_{1}$ & $\mathrm{~S}(20 \mathrm{Kg} / \mathrm{ha}) \mathrm{G}(100 \mathrm{ppm}) \mathrm{N}(50 \mathrm{ppm})$ & 59.17 & 61.17 & 60.17 & 12.27 & 12.77 & 12.52 & 1.42 & 1.72 & 1.57 & 1.30 & 1.60 & 1.45 \\
\hline $\mathrm{S}_{1} \mathrm{G}_{2} \mathrm{~N}_{2}$ & $\mathrm{~S}(20 \mathrm{Kg} / \mathrm{ha}) \mathrm{G}(100 \mathrm{ppm}) \mathrm{N}(100 \mathrm{ppm})$ & 59.17 & 61.17 & 60.17 & 12.40 & 12.90 & 12.65 & 1.44 & 1.74 & 1.59 & 1.33 & 1.63 & 1.48 \\
\hline $\mathbf{S}_{2} \mathbf{G}_{0} \mathbf{N}_{0}$ & $\mathrm{~S}(40 \mathrm{Kg} / \mathrm{ha}) \mathrm{G}(0 \mathrm{ppm}) \mathrm{N}(0 \mathrm{ppm})$ & 55.43 & 57.43 & 56.43 & 11.60 & 12.10 & 11.85 & 1.29 & 1.59 & 1.44 & 1.17 & 1.47 & 1.32 \\
\hline $\mathbf{S}_{2} \mathbf{G}_{0} \mathbf{N}_{1}$ & $\mathrm{~S}(40 \mathrm{Kg} / \mathrm{ha}) \mathrm{G}(0 \mathrm{ppm}) \mathrm{N}(50 \mathrm{ppm})$ & 60.07 & 62.40 & 61.23 & 12.73 & 13.32 & 13.03 & 1.48 & 1.87 & 1.68 & 1.36 & 1.74 & 1.55 \\
\hline $\mathbf{S}_{2} \mathbf{G}_{0} \mathbf{N}_{2}$ & $\mathrm{~S}(40 \mathrm{Kg} / \mathrm{ha}) \mathrm{G}(0 \mathrm{ppm}) \mathrm{N}(100 \mathrm{ppm})$ & 60.37 & 62.87 & 61.62 & 12.80 & 13.47 & 13.13 & 1.50 & 1.96 & 1.73 & 1.37 & 1.83 & 1.60 \\
\hline$S_{2} G_{1} N_{0}$ & $\mathrm{~S}(40 \mathrm{Kg} / \mathrm{ha}) \mathrm{G}(50 \mathrm{ppm}) \mathrm{N}(0 \mathrm{ppm})$ & 58.27 & 60.27 & 59.27 & 11.83 & 12.33 & 12.08 & 1.34 & 1.64 & 1.49 & 1.28 & 1.58 & 1.43 \\
\hline $\mathrm{S}_{2} \mathbf{G}_{1} \mathbf{N}_{1}$ & $\mathrm{~S}(40 \mathrm{Kg} / \mathrm{ha}) \mathrm{G}(50 \mathrm{ppm}) \mathrm{N}(50 \mathrm{ppm})$ & 61.93 & 64.93 & 63.43 & 13.33 & 14.25 & & 1.55 & 2.26 & 1.91 & 1.46 & 2.18 & 1.82 \\
\hline $\mathbf{S}_{2} \mathbf{G}_{1} \mathbf{N}_{2}$ & $\mathrm{~S}(40 \mathrm{Kg} / \mathrm{ha}) \mathrm{G}(50 \mathrm{ppm}) \mathrm{N}(100 \mathrm{ppm})$ & 63.60 & 66.77 & 65.18 & 13.43 & 14.43 & 13.93 & 1.57 & 2.37 & 1.97 & 1.51 & 2.31 & 1.91 \\
\hline $\mathbf{S}_{2} \mathbf{G}_{2} \mathbf{N}_{0}$ & $\mathrm{~S}(40 \mathrm{Kg} / \mathrm{ha}) \mathrm{G}(100 \mathrm{ppm}) \mathrm{N}(0 \mathrm{ppm})$ & 57.13 & 59.13 & 58.13 & 11.80 & 12.30 & 12.05 & 1.33 & 1.63 & 1.48 & 1.27 & 1.57 & 1.42 \\
\hline $\mathbf{S}_{2} \mathbf{G}_{2} \mathbf{N}_{1}$ & $\mathrm{~S}(40 \mathrm{Kg} / \mathrm{ha}) \mathrm{G}(100 \mathrm{ppm}) \mathrm{N}(50 \mathrm{ppm})$ & 61.60 & 64.27 & 62.93 & 13.10 & 13.85 & 13.48 & 1.52 & 2.52 & 2.02 & 1.39 & 1.94 & 1.67 \\
\hline \multirow[t]{3}{*}{$\mathbf{S}_{2} \mathbf{G}_{2} \mathbf{N}_{2}$} & $\mathrm{~S}(40 \mathrm{Kg} / \mathrm{ha}) \mathrm{G}(100 \mathrm{ppm}) \mathrm{N}(100 \mathrm{ppm})$ & 61.67 & 64.50 & 63.08 & 13.13 & 13.97 & 13.55 & 1.53 & 2.16 & 1.84 & 1.41 & 2.04 & 1.72 \\
\hline & S.Em \pm & 1.25 & 0.69 & 0.64 & 0.20 & 0.16 & 0.12 & 0.06 & 0.06 & 0.05 & 0.04 & 0.06 & 0.04 \\
\hline & C.D. (P 0.05) level & N.S. & N.S. & N.S. & N.S. & N.S. & N.S. & N.S. & 0.16 & N.S. & N.S. & N.S. & N.S. \\
\hline
\end{tabular}


Table.3 Effect of different doses of Sulphur, $\mathrm{GA}_{3}$ and NAA on yield traits and yield

\begin{tabular}{|c|c|c|c|c|c|c|c|c|c|c|c|c|c|}
\hline \multirow[t]{2}{*}{$\begin{array}{l}\text { Treat. } \\
\text { Symb. }\end{array}$} & \multirow[t]{2}{*}{ Treatments } & \multicolumn{3}{|c|}{$\begin{array}{c}\text { Fresh weight of bulb } \\
\text { (g) }\end{array}$} & \multicolumn{3}{|c|}{$\begin{array}{l}\text { Polar diameter of } \\
\text { bulb }(\mathbf{c m})\end{array}$} & \multicolumn{3}{|c|}{$\begin{array}{c}\text { Equatorial diameter of } \\
\text { bulb }(\mathrm{cm})\end{array}$} & \multicolumn{3}{|c|}{ Bulb yield ha ${ }^{-1}(q)$} \\
\hline & & $\begin{array}{c}1^{\text {st }} \\
\text { Year }\end{array}$ & $\begin{array}{c}2^{\text {nd }} \\
\text { Year }\end{array}$ & Pooled & $\begin{array}{c}1^{\text {st }} \\
\text { Year }\end{array}$ & $\begin{array}{c}2^{\text {nd }} \\
\text { Year }\end{array}$ & Pooled & $\begin{array}{c}1^{\text {st }} \\
\text { Year }\end{array}$ & $\begin{array}{l}2^{\text {nd }} \\
\text { Year }\end{array}$ & Pooled & $\begin{array}{c}1^{\text {st }} \\
\text { Year }\end{array}$ & $\begin{array}{c}2^{\text {nd }} \\
\text { Year }\end{array}$ & Pooled \\
\hline $\mathbf{S}_{\mathbf{0}}$ & Sulphur (0 kg/ha) & 74.32 & 79.36 & 76.84 & 5.23 & 5.53 & 5.38 & 4.35 & 4.65 & 4.50 & 198.17 & 189.17 & 193.67 \\
\hline $\mathbf{S}_{1}$ & Sulphur (20 kg/ha) & 85.33 & 90.37 & 87.85 & 5.70 & 6.00 & 5.85 & 4.73 & 5.03 & 4.88 & 230.56 & 220.96 & 225.76 \\
\hline \multirow[t]{3}{*}{$\mathbf{S}_{2}$} & Sulphur (40 kg/ha) & 90.93 & 96.46 & 93.69 & 5.89 & 6.39 & 6.14 & 4.82 & 5.26 & 5.04 & 244.01 & 240.31 & 242.16 \\
\hline & S.Em \pm & 0.09 & 0.10 & 0.06 & 0.01 & 0.01 & 0.01 & 0.01 & 0.01 & 0.01 & 0.46 & 0.65 & 0.48 \\
\hline & C.D. (P 0.05) level & 0.26 & 0.30 & 0.18 & 0.02 & 0.02 & 0.03 & 0.02 & 0.03 & 0.03 & 1.32 & 1.85 & 1.33 \\
\hline $\mathbf{G}_{\mathbf{0}}$ & $\mathrm{GA}_{3}(0 \mathrm{PPM})$ & 81.25 & 86.46 & 83.85 & 5.52 & 5.85 & 5.69 & 4.56 & 4.89 & 4.73 & 216.73 & 208.62 & 212.67 \\
\hline $\mathbf{G}_{1}$ & $\mathrm{GA}_{3}(50 \mathrm{PPM})$ & 85.69 & 90.93 & 88.31 & 5.69 & 6.09 & 5.89 & 4.69 & 5.11 & 4.90 & 231.01 & 224.53 & 227.77 \\
\hline \multirow[t]{3}{*}{$\mathbf{G}_{2}$} & $\mathrm{GA}_{3}(100 \mathrm{PPM})$ & 83.64 & 88.80 & 86.22 & 5.61 & 5.98 & 5.80 & 4.64 & 4.94 & 4.79 & 225.00 & 217.30 & 221.15 \\
\hline & S.Em \pm & 0.09 & 0.10 & 0.06 & 0.01 & 0.01 & 0.01 & 0.01 & 0.01 & 0.01 & 0.46 & 0.65 & 0.48 \\
\hline & C.D. (P 0.05) level & 0.26 & 0.30 & 0.18 & 0.02 & 0.02 & 0.03 & 0.02 & 0.03 & 0.03 & 1.32 & 1.85 & 1.33 \\
\hline $\mathbf{N}_{0}$ & NAA (0 PPM) & 78.11 & 83.11 & 80.61 & 5.41 & 5.71 & 5.56 & 4.51 & 4.84 & 4.68 & 206.28 & 196.60 & 201.44 \\
\hline $\mathbf{N}_{1}$ & NAA (50 PPM) & 85.74 & 91.07 & 88.41 & 5.69 & 6.07 & 5.88 & 4.67 & 5.02 & 4.85 & 230.82 & 224.69 & 227.76 \\
\hline \multirow[t]{3}{*}{$\mathbf{N}_{2}$} & NAA (100 PPM) & 86.73 & 92.01 & 89.37 & 5.73 & 6.14 & 5.94 & 4.70 & 5.08 & 4.89 & 235.63 & 229.16 & 232.40 \\
\hline & S.Em \pm & 0.09 & 0.10 & 0.06 & 0.01 & 0.01 & 0.01 & 0.01 & 0.01 & 0.01 & 0.46 & 0.65 & 0.48 \\
\hline & C.D. (P 0.05) level & 0.26 & 0.30 & 0.18 & 0.02 & 0.02 & 0.03 & 0.02 & 0.03 & 0.03 & 1.32 & 1.85 & 1.33 \\
\hline
\end{tabular}


Table.4 Interaction effect of different doses of sulphur, $\mathrm{GA}_{3}$ and NAA on yield traits and yield

\begin{tabular}{|c|c|c|c|c|c|c|c|c|c|c|c|c|c|}
\hline \multirow[t]{2}{*}{$\begin{array}{l}\text { reat. } \\
\text { Symb. }\end{array}$} & \multirow[t]{2}{*}{ Treatments } & \multicolumn{3}{|c|}{ Fresh weight of bulb (g) } & \multicolumn{3}{|c|}{$\begin{array}{c}\text { Polar diameter of bulb } \\
(\mathbf{c m})\end{array}$} & \multicolumn{3}{|c|}{$\begin{array}{l}\text { Equatorial diameter of bult } \\
(\mathbf{c m})\end{array}$} & \multicolumn{3}{|c|}{ Bulb yield ha $^{-1}(q)$} \\
\hline & & $1^{\text {st }}$ Year & $2^{\text {nd }}$ Year & Pooled & $1^{\text {st }}$ Year & $2^{\text {nd }}$ Year & Pooled & $1^{\text {st }}$ Year & $2^{\text {nd }}$ Year & Pooled & $1^{\text {st }}$ Year & $2^{\text {nd }}$ Year & Pooled \\
\hline $\mathbf{S}_{0} \mathbf{G}_{0} \mathbf{N}_{0}$ & $\mathrm{~S}(0 \mathrm{Kg} / \mathrm{ha}) \mathrm{G}(0 \mathrm{ppm}) \mathrm{N}(0 \mathrm{ppm})$ & 70.00 & 75.00 & 72.50 & 5.05 & 5.35 & 5.20 & 4.12 & 4.42 & 4.27 & 179.50 & 169.91 & 174.71 \\
\hline $\mathrm{S}_{0} \mathrm{G}_{0} \mathrm{~N}_{1}$ & $\mathrm{~S}(0 \mathrm{Kg} / \mathrm{ha}) \mathrm{G}(0 \mathrm{ppm}) \mathrm{N}(50 \mathrm{ppm})$ & 73.70 & 79.03 & 76.37 & 5.21 & 5.51 & 5.36 & 4.30 & 4.60 & 4.45 & 192.81 & 183.21 & 188.01 \\
\hline $\mathrm{S}_{0} \mathrm{G}_{0} \mathrm{~N}_{2}$ & $\mathrm{~S}(0 \mathrm{Kg} / \mathrm{ha}) \mathrm{G}(0 \mathrm{ppm}) \mathrm{N}(100 \mathrm{ppm})$ & 74.57 & 79.57 & 77.07 & 5.25 & 5.55 & 5.40 & 4.34 & 4.64 & 4.49 & 199.67 & 190.06 & 194.87 \\
\hline $\mathbf{S}_{0} \mathbf{G}_{1} \mathbf{N}_{0}$ & $\mathrm{~S}(0 \mathrm{Kg} / \mathrm{ha}) \mathrm{G}(50 \mathrm{ppm}) \mathrm{N}(0 \mathrm{ppm})$ & 73.37 & 78.37 & 75.87 & 5.17 & 5.47 & 5.32 & 4.26 & 4.56 & 4.41 & 185.95 & 177.04 & 181.50 \\
\hline $\mathbf{S}_{0} \mathbf{G}_{1} \mathbf{N}_{1}$ & $\mathrm{~S}(0 \mathrm{Kg} / \mathrm{ha}) \mathrm{G}(50 \mathrm{ppm}) \mathrm{N}(50 \mathrm{ppm})$ & 76.77 & 81.77 & 79.27 & 5.33 & 5.63 & 5.48 & 4.49 & 4.79 & 4.64 & 213.65 & 205.42 & 209.54 \\
\hline $\mathbf{S}_{0} \mathbf{G}_{1} \mathbf{N}_{2}$ & $\mathrm{~S}(0 \mathrm{Kg} / \mathrm{ha}) \mathrm{G}(50 \mathrm{ppm}) \mathrm{N}(100 \mathrm{ppm})$ & 77.77 & 82.77 & 80.27 & 5.36 & 5.66 & 5.51 & 4.55 & 4.85 & 4.70 & 214.62 & 206.39 & 210.50 \\
\hline $\mathbf{S}_{0} \mathbf{G}_{2} \mathbf{N}_{0}$ & $\mathrm{~S}(0 \mathrm{Kg} / \mathrm{ha}) \mathrm{G}(100 \mathrm{ppm}) \mathrm{N}(0 \mathrm{ppm})$ & 72.23 & 77.23 & 74.73 & 5.12 & 5.42 & 5.27 & 4.22 & 4.52 & 4.37 & 182.53 & 173.61 & 178.07 \\
\hline $\mathbf{S}_{0} \mathbf{G}_{2} \mathbf{N}_{1}$ & $\mathrm{~S}(0 \mathrm{Kg} / \mathrm{ha}) \mathrm{G}(100 \mathrm{ppm}) \mathrm{N}(50 \mathrm{ppm})$ & 74.93 & 79.93 & 77.43 & 5.28 & 5.58 & 5.43 & 4.39 & 4.69 & 4.54 & 205.84 & 196.92 & 201.38 \\
\hline $\mathbf{S}_{0} \mathbf{G}_{2} \mathbf{N}_{2}$ & $\mathrm{~S}(0 \mathrm{Kg} / \mathrm{ha}) \mathrm{G}(100 \mathrm{ppm}) \mathrm{N}(100 \mathrm{ppm})$ & 75.57 & 80.57 & 78.07 & 5.31 & 5.61 & 5.46 & 4.46 & 4.76 & 4.61 & 208.92 & 200.01 & 204.47 \\
\hline $\mathbf{S}_{1} \mathbf{G}_{0} \mathbf{N}_{0}$ & $\mathrm{~S}(20 \mathrm{Kg} / \mathrm{ha}) \mathrm{G}(0 \mathrm{ppm}) \mathrm{N}(0 \mathrm{ppm})$ & 78.73 & 83.73 & 81.23 & 5.41 & 5.71 & 5.56 & 4.55 & 4.85 & 4.70 & 215.02 & 204.74 & 209.88 \\
\hline $\mathbf{S}_{1} \mathbf{G}_{0} \mathbf{N}_{1}$ & $\mathrm{~S}(20 \mathrm{Kg} / \mathrm{ha}) \mathrm{G}(0 \mathrm{ppm}) \mathrm{N}(50 \mathrm{ppm})$ & 85.27 & 90.60 & 87.93 & 5.77 & 6.07 & 5.92 & 4.73 & 5.03 & 4.88 & 228.29 & 218.01 & 223.15 \\
\hline $\mathrm{S}_{1} \mathrm{G}_{0} \mathrm{~N}_{2}$ & $\mathrm{~S}(20 \mathrm{Kg} / \mathrm{ha}) \mathrm{G}(0 \mathrm{ppm}) \mathrm{N}(100 \mathrm{ppm})$ & 86.67 & 91.67 & 89.17 & 5.77 & 6.07 & 5.92 & 4.73 & 5.03 & 4.88 & 234.23 & 223.94 & 229.08 \\
\hline $\mathbf{S}_{1} \mathbf{G}_{1} \mathbf{N}_{\mathbf{0}}$ & $\mathrm{S}(20 \mathrm{Kg} / \mathrm{ha}) \mathrm{G}(50 \mathrm{ppm}) \mathrm{N}(0 \mathrm{ppm})$ & 81.33 & 86.33 & 83.83 & 5.59 & 5.89 & 5.74 & 4.70 & 5.00 & 4.85 & 217.08 & 208.17 & 212.63 \\
\hline $\mathbf{S}_{1} \mathbf{G}_{1} \mathbf{N}_{1}$ & $\mathrm{~S}(20 \mathrm{Kg} / \mathrm{ha}) \mathrm{G}(50 \mathrm{ppm}) \mathrm{N}(50 \mathrm{ppm})$ & 90.00 & 95.00 & 92.50 & 5.83 & 6.13 & 5.98 & 4.81 & 5.11 & 4.96 & 243.00 & 234.09 & 238.54 \\
\hline $\mathbf{S}_{1} \mathbf{G}_{1} \mathbf{N}_{2}$ & $\mathrm{~S}(20 \mathrm{Kg} / \mathrm{ha}) \mathrm{G}(50 \mathrm{ppm}) \mathrm{N}(100 \mathrm{ppm})$ & 90.67 & 95.67 & 93.17 & 5.89 & 6.19 & 6.04 & 4.81 & 5.11 & 4.96 & 243.69 & 234.77 & 239.23 \\
\hline $\mathbf{S}_{1} \mathbf{G}_{2} \mathbf{N}_{0}$ & $\mathrm{~S}(20 \mathrm{Kg} / \mathrm{ha}) \mathrm{G}(100 \mathrm{ppm}) \mathrm{N}(0 \mathrm{ppm})$ & 80.00 & 85.00 & 82.50 & 5.50 & 5.80 & 5.65 & 4.69 & 4.99 & 4.84 & 216.40 & 206.80 & 211.60 \\
\hline $\mathbf{S}_{1} \mathbf{G}_{2} \mathbf{N}_{1}$ & $\mathrm{~S}(20 \mathrm{Kg} / \mathrm{ha}) \mathrm{G}(100 \mathrm{ppm}) \mathrm{N}(50 \mathrm{ppm})$ & 87.33 & 92.33 & 89.83 & 5.77 & 6.07 & 5.92 & 4.76 & 5.06 & 4.91 & 234.50 & 224.90 & 229.70 \\
\hline $\mathbf{S}_{1} \mathbf{G}_{2} \mathbf{N}_{2}$ & $\mathrm{~S}(20 \mathrm{Kg} / \mathrm{ha}) \mathrm{G}(100 \mathrm{ppm}) \mathrm{N}(100 \mathrm{ppm})$ & 88.00 & 93.00 & 90.50 & 5.80 & 6.10 & 5.95 & 4.77 & 5.07 & 4.92 & 242.86 & 233.26 & 238.06 \\
\hline $\mathbf{S}_{2} \mathbf{G}_{0} \mathbf{N}_{0}$ & $\mathrm{~S}(40 \mathrm{Kg} / \mathrm{ha}) \mathrm{G}(0 \mathrm{ppm}) \mathrm{N}(0 \mathrm{ppm})$ & 79.33 & 84.33 & 81.83 & 5.43 & 5.73 & 5.58 & 4.63 & 4.93 & 4.78 & 215.57 & 205.29 & 210.43 \\
\hline $\mathbf{S}_{2} \mathbf{G}_{0} \mathbf{N}_{1}$ & $\mathrm{~S}(40 \mathrm{Kg} / \mathrm{ha}) \mathrm{G}(0 \mathrm{ppm}) \mathrm{N}(50 \mathrm{ppm})$ & 91.00 & 96.67 & 93.83 & 5.91 & 6.29 & 6.10 & 4.81 & 5.19 & 5.00 & 241.77 & 240.08 & 240.92 \\
\hline $\mathbf{S}_{2} \mathbf{G}_{0} \mathbf{N}_{2}$ & $\mathrm{~S}(40 \mathrm{Kg} / \mathrm{ha}) \mathrm{G}(0 \mathrm{ppm}) \mathrm{N}(100 \mathrm{ppm})$ & 92.00 & 97.50 & 94.75 & 5.91 & 6.37 & 6.14 & 4.85 & 5.32 & 5.09 & 243.69 & 242.31 & 243.00 \\
\hline $\mathbf{S}_{2} \mathbf{G}_{1} \mathbf{N}_{0}$ & $\mathrm{~S}(40 \mathrm{Kg} / \mathrm{ha}) \mathrm{G}(50 \mathrm{ppm}) \mathrm{N}(0 \mathrm{ppm})$ & 84.67 & 89.67 & 87.17 & 5.75 & 6.05 & 5.90 & 4.73 & 5.28 & 5.00 & 223.94 & 213.65 & 218.80 \\
\hline $\mathbf{S}_{2} \mathbf{G}_{1} \mathbf{N}_{1}$ & $\mathrm{~S}(40 \mathrm{Kg} / \mathrm{ha}) \mathrm{G}(50 \mathrm{ppm}) \mathrm{N}(50 \mathrm{ppm})$ & 98.00 & 104.00 & 101.00 & 6.11 & 6.83 & 6.47 & 4.90 & 5.53 & 5.22 & 266.18 & 264.81 & 265.49 \\
\hline $\mathbf{S}_{2} \mathbf{G}_{1} \mathbf{N}_{2}$ & $\mathrm{~S}(40 \mathrm{Kg} / \mathrm{ha}) \mathrm{G}(50 \mathrm{ppm}) \mathrm{N}(100 \mathrm{ppm})$ & 98.67 & 104.83 & 101.75 & 6.17 & 6.97 & 6.57 & 4.93 & 5.73 & 5.33 & 270.97 & 276.46 & 273.72 \\
\hline $\mathbf{S}_{2} \mathbf{G}_{2} \mathbf{N}_{\mathbf{0}}$ & $\mathrm{S}(40 \mathrm{Kg} / \mathrm{ha}) \mathrm{G}(100 \mathrm{ppm}) \mathrm{N}(0 \mathrm{ppm})$ & 83.33 & 88.33 & 85.83 & 5.67 & 5.97 & 5.82 & 4.72 & 5.02 & 4.87 & 220.51 & 210.23 & 215.37 \\
\hline $\mathbf{S}_{2} \mathbf{G}_{2} \mathbf{N}_{1}$ & $\mathrm{~S}(40 \mathrm{Kg} / \mathrm{ha}) \mathrm{G}(100 \mathrm{ppm}) \mathrm{N}(50 \mathrm{ppm})$ & 94.67 & 100.33 & 97.50 & 5.99 & 6.54 & 6.26 & 4.87 & 5.17 & 5.02 & 251.37 & 254.80 & 253.08 \\
\hline \multirow[t]{3}{*}{$\mathbf{S}_{2} \mathbf{G}_{2} \mathbf{N}_{2}$} & $\mathrm{~S}(40 \mathrm{Kg} / \mathrm{ha}) \mathrm{G}(100 \mathrm{ppm}) \mathrm{N}(100 \mathrm{ppm})$ & 96.67 & 102.50 & 99.58 & 6.10 & 6.73 & 6.42 & 4.89 & 5.19 & 5.04 & 262.06 & 255.20 & 258.63 \\
\hline & S.Em \pm & 0.27 & 0.31 & 0.20 & 0.01 & 0.02 & 0.03 & 0.01 & 0.03 & 0.03 & 1.39 & 1.94 & 1.44 \\
\hline & C.D. (P 0.05) level & 0.78 & 0.91 & 0.57 & 0.04 & 0.05 & 0.09 & 0.04 & 0.08 & 0.09 & 3.95 & 5.52 & 4.00 \\
\hline
\end{tabular}


Foliar application of NAA @ 100 PPM $\left(\mathrm{N}_{2}\right)$ resulted in significantly maximum 235.63, 229.16 and $232.40 \mathrm{q} / \mathrm{ha}$ bulb yield followed by $\mathrm{N}_{1}$ (NAA @ $50 \mathrm{ppm}$ ) while, it was observed lowest 206.28, 196.60 and 201.44 $\mathrm{q} / \mathrm{ha}$ in treatment $\mathrm{N}_{0}$ (NAA 0 PPM) at first year, second year and in pooled, respectively. The results of the present investigation are in accordance with the observations of Bose et al., (2009), Singh et al., (2014), Pratap et al., (2017) and Meena et al., (2017). The increase in the fresh weight of bulb, polar and equatorial diameter of bulb and bulb yield mainly attributed to bigger bulb formation, more number of leaves, higher dry matter accumulation. Manipulation of source (leaf) and sink (bulb) relationship through the above treatments may be the principal reason for yield improvement. Higher yield in onion has so far been achieved mainly through the judicious applications of various plant growth regulators.

Interaction effect of Sulphur, $\mathrm{GA}_{3}$ and NAA on yield and yield parameters

It is obvious from the Table 4 that the significantly maximum 98.67, 104.83 and $101.75 \mathrm{~g}$ fresh weight of bulb were recorded in treatment combination $\mathrm{S}_{2} \mathrm{G}_{1} \mathrm{~N}_{2}$ (S 40 $\mathrm{Kg} / \mathrm{ha}+\mathrm{GA}_{3}$ 50ppm+ NAA100ppm) followed by $\mathrm{S}_{2} \mathrm{G}_{1} \mathrm{~N}_{1} \quad\left(\mathrm{~S} 40 \mathrm{Kg} / \mathrm{ha}+\mathrm{GA}_{3}\right.$ 50ppm+ NAA50ppm) (98.0, 104.0 and $101.0 \mathrm{~g}$ ), while, it was recorded lowest 70.0, 75.0 and $72.50 \mathrm{~g}$ in treatment $\mathrm{S}_{0} \mathrm{G}_{0} \mathrm{~N}_{0}\left(\mathrm{~S} 0 \mathrm{Kg} / \mathrm{ha}+\mathrm{GA}_{3}\right.$ Oppm+ NAA 0ppm) at first year, second year and in pooled, respectively. Similarly the significantly maximum $6.17,6.97$ and 6.57 $\mathrm{cm}$ polar diameter of bulb were recorded in treatment combination $\mathrm{S}_{2} \mathrm{G}_{1} \mathrm{~N}_{2}(\mathrm{~S} 40 \mathrm{Kg} / \mathrm{ha}+$ $\mathrm{GA}_{3}$ 50ppm+ NAA100ppm) followed by $\mathrm{S}_{2} \mathrm{G}_{1} \mathrm{~N}_{1} \quad$ (S $40 \mathrm{Kg} / \mathrm{ha}+\mathrm{GA}_{3} \quad 50 \mathrm{ppm}+$ NAA50ppm) $(6.11,6.83$ and $6.47 \mathrm{~cm})$, while, it was recorded lowest $5.05,5.35$ and $5.20 \mathrm{~cm}$ in treatment $\mathrm{S}_{0} \mathrm{G}_{0} \mathrm{~N}_{0}\left(\mathrm{~S} 0 \mathrm{Kg} / \mathrm{ha}+\mathrm{GA}_{3} 0 \mathrm{ppm}+\right.$ NAA 0ppm) at first year, second year and in pooled, respectively. Results of the present investigation are also in confirmatory with the findings of Rashid (2010). Significantly maximum 4.93, 5.73 and $5.33 \mathrm{~cm}$ equatorial diameter of bulb were recorded in treatment combination $\mathrm{S}_{2} \mathrm{G}_{1} \mathrm{~N}_{2}$ (S $40 \mathrm{Kg} / \mathrm{ha}+\mathrm{GA}_{3}$ 50ppm+ NAA100ppm) followed by $\mathrm{S}_{2} \mathrm{G}_{1} \mathrm{~N}_{1}$ (S $40 \mathrm{Kg} / \mathrm{ha}+\mathrm{GA}_{3} 50 \mathrm{ppm}+\mathrm{NAA} 50 \mathrm{ppm}$ ) (4.90, 5.53 and $5.22 \mathrm{~cm}$ ), while, it was recorded lowest $4.12,4.42$ and $4.27 \mathrm{~cm}$ in treatment $\mathrm{S}_{0} \mathrm{G}_{0} \mathrm{~N}_{0}\left(\mathrm{~S} 0 \mathrm{Kg} / \mathrm{ha}+\mathrm{GA}_{3} 0 \mathrm{ppm}+\right.$ NAA 0ppm) at first year, second year and in pooled, respectively. Significantly maximum 270.97, 276.46 and $273.72 \mathrm{q} /$ ha bulb yield were recorded in treatment combination $\mathrm{S}_{2} \mathrm{G}_{1} \mathrm{~N}_{2} \quad$ (S $40 \mathrm{Kg} / \mathrm{ha}+\mathrm{GA}_{3} \quad 50 \mathrm{ppm}+$ NAA100ppm) followed by $\mathrm{S}_{2} \mathrm{G}_{1} \mathrm{~N}_{1}$ (S 40 $\mathrm{Kg} / \mathrm{ha}+\mathrm{GA}_{3}$ 50ppm+ NAA50ppm) (266.18, 264.81 and $265.49 \mathrm{q} / \mathrm{ha}$ ), while, it was recorded lowest $179.50,169.91$ and 174.71 $\mathrm{q} / \mathrm{ha}$ in treatment $\mathrm{S}_{0} \mathrm{G}_{0} \mathrm{~N}_{0}\left(\mathrm{~S} 0 \mathrm{Kg} / \mathrm{ha}+\mathrm{GA}_{3}\right.$ Oppm+ NAA 0ppm) at first, second year and pooled, respectively. The results of the present investigation are in accordance with the findings of Rashid (2010), Sitapara et al., (2011), Trivedi and Dhumal (2017) and Meena et al., (2017). The increase in the fresh weight of bulb, polar and equatorial diameter of bulb and bulb yield mainly attributed to more number of leaves, higher dry matter accumulation. Manipulation of source (leaf) and sink (bulb) relationship through the above treatments may be the principal reason for yield improvement. Higher yield in onion has so far been achieved mainly through the judicious applications of various plant growth regulators and sulphur.

\section{References}

Bose, U.S., Bisen, A., Sharma, R. K. and Dongre, R. (2009). Effect of micro nutrients along with growth regulator on growth and yield of onion. International Journal of Applied Agricultural Research. 4 (3): 267-271. 
El Sayed, Hameda; Abdullah, E. A., ElMorsy, H. A. and Hanan Saleh Al Othaimen (2015). The role of sulphur and certain foliar spray levels of micronutrients on garlic (Allium sativum L.) plant. Int. J. Curr. Res. Biosci. Plant Biol. 2 (6): 76-87.

Govind, S., Maji, R., Kumawat, A., Pal, S. Kumar and Saha, S. (2015). Improvement of growth, yield and quality of garlic (Allium sativum L.) CV. G-282 through a novel approach. Bio Science. 10 (1): 23-27.

Jain, Goldi; Kushwah, S. S., Singh, O. P. and Verma, K. S. (2014). Effect of different doses of nitrogen and sulphur on growth, yield and quality of onion (Allium cepa). The Indian Journal of Agricultural Sciences. 84 (11): 205-208.

Meena, Rajesh Kumar; Dhaka, R. S., Meena, Narender Kumar and Meena, Sunil (2017). Effect of foliar application of NAA and $\mathrm{GA}_{3}$ on growth and yield of okra [Abelmoschus esculentus (L.) Moench] cv. Arka Anamika. Int. J. Pure App. Biosci. 5 (2): 1057-1062.

Rashid, M. H. A. (2010). Effect of sulphur and $\mathrm{GA}_{3}$ on the growth and yield of onion. Progress. Agric. 21 (1\& 2): 57 63.

Singh, Harsh Deep; Maji, Sutanu and Sanjay
Kumar (2014). Influence of plant bioregulators on growth and yield of garlic (Allium sativum L.). International Journal of Agricultural Sciences. 10 (2): 546-549.

Sitapara, H. H., Vihol, N. J., Patel, M. J. and Patel, J. S. (2011). Effect of growth regulators and micro nutrient on growth and yield of cauliflower cv. 'SNOWBALL-16'. The Asian Journal of Horticulture. 6 (2): 348 - 351.

Suman Smriti; Rajesh Kumar and Singh, S. K. (2002). Effect of sulphur and boron nutrition on growth, yield and quality of onion (Allium cepa L.). Journal of Applied Biology. 12 (1/2): 40-46.

Trivedi, Ashwin and Dhumal, K.N. (2017). Effect of micronutrients, growth regulators and organic manures on yield biochemical and mineral component of onion (Allium cepa L.) grown in vertisols. Int. J. Curr. Microbiology and Applied Sciences. 6 (5): 1759-1771.

Yadagiri, Jagati; Gupta, Prashant Kumar; Tiwari, Rajesh and Singh, V. B. (2017). Improvement of growth and yield of onion (Allium cepa L.) cv. Agrifound Light Red through different application methods of gibberellic acid and Trichoderma viride. Int. J. Pure App. Biosci. 5 (4): 1444-1450.

\section{How to cite this article:}

Lal Singh, A.K. Barholia, Rashmi Bajpai, N.S. Bhadauria and Singh, V.B. 2019. Response of Kharif Onion (Allium cepa L.) for Growth and Yield to Different Doses of Sulphur, $\mathrm{GA}_{3}$ and NAA. Int.J.Curr.Microbiol.App.Sci. 8(01): 2362-2372. doi: https://doi.org/10.20546/ijcmas.2019.801.248 